\title{
Defining turn taking in intervention for young children with autism: A review of the literature
}

\author{
Kwangwon Lee', Ashley Staggs ${ }^{2}$
}

\begin{abstract}
Turn taking is a form of preverbal, dyadic, reciprocal communication that may support key areas of development, such as language and joint attention, and may serve different functions depending on each communicative partner's intent. As such, it has been incorporated in interventions targeting various outcomes in young children with autism. However, there is inconsistency in how researchers define turn taking and explorations on how turn taking is defined across these interventions have not yet been reported in the current literature. Therefore, the purpose of this review was to investigate how turn taking is operationally defined based on communicative intent in the current literature on interventions for young children with autism and to explore additional intervention content to provide fuller context to how turn taking has been promoted. A search was conducted across databases to identify intervention studies for young children with autism that incorporated an embedded turn-taking component. Peer-reviewed articles were then coded based on turn-taking communicative intent, and additional intervention content was categorized. Findings across 14 studies indicate variability among turn-taking definitions both in communicative function and form. The results also reveal that turn taking has been promoted through different intervention approaches that incorporate diverse agents, settings, and methodology. Researchers and practitioners should consider specificity and clarity when defining turn taking to most optimally meet the developmental needs of young children with autism in future interventions.
\end{abstract}

\section{Article History}

Received: 22 May 2021

Accepted: 02 July 2021

\section{Keywords}

Autism; Early childhood; Intervention; Preverbal

communication; Turn taking

\section{Introduction}

Children with autism often display core challenges in social communication and restrictive repetitive behaviors (RRBs) that may be present throughout the lifespan (American Psychiatric Association [APA], 2013; Gillespie-Lynch et al., 2012; Moriuchi, Klin, \& Jones, 2016). Symptom severity levels vary among individuals with autism, who may have symptoms ranging from mild to more severe manifestations that may require varying degrees of support (APA, 2013; Kim et al., 2019). The most recent report from the Centers for Disease Control and Prevention estimates that 1 in 54 children in the United States has autism, a prevalence rate that has been increasing since 2002 (Maenner et al., 2020). As the prevalence rate increases, so too does the need for developing early interventions that support the unique needs of young children with autism.

For many young children with autism, the core challenge in social communication is evident early in life, before children with typical development begin using verbal communication (Moriuchi et al., 2016; Poon, Watson, Baranek, \& Poe, 2012), and is often one of the first indicators that a child has autism (Curcio, 1978; Mundy, 2016). Infants who later receive an autism diagnosis have been observed exhibiting challenges in preverbal forms of social communication, such as eye gaze and head orienting to parents' bids for attention (Moriuchi et al., 2016; Poon et al., 2012). These challenges may become more distinct as children progress in development, when important milestones that are seen in children with typical development, such as competency in joint attention and use of expressive and receptive language, are not

\footnotetext{
${ }^{1}$ Eastern Connecticut State University, Education Department, Willimantic, CT, USA, e-mail: leekw@easternct.edu, ORCID: $\underline{\text { https://orcid.org/0000-0001-7956-1895 }}$

${ }^{2}$ Russellville Independent School District, Administration, Russellville, KY, USA, ashley.staggs@russellville.kyschools.us, ORCID: https://orcid.org/0000-0001-8927-454X
} 
reached (e.g., Delehanty, Stronach, Guthrie, Slate, \& Wetherby, 2018; Mundy, Sigman, Ungerer, \& Sherman, 1986).

\section{Preverbal Turn Taking in Intervention}

To support their social communication needs, various interventions have been developed that promote outcomes in young children with autism who require support in key areas, such as play, relationships, and language (e.g., Greenspan, \& Wieder, 2006; Rogers, Dawson, \& Vismara, 2012). A commonly embedded intervention component is turn taking, a form of dyadic, reciprocal, preverbal communication that children begin using early in life with other people (Clifford \& Dissanayake, 2009; Schertz, Odom, Baggett, \& Sideris, 2018). An example of turn taking might be observed when a child is stacking blocks on a playroom floor and their parent sits next to them, stacks a block, and then allows the child to take a turn stacking another block. In this example, the child and their parent are engaging in a simple, back-and-forth turn-taking exchange centered around a certain activity. Turn taking may have important implications in supporting later developmental outcomes, and some researchers theorize that dyadic, preverbal turn taking may help children develop competency in more complex, triadic forms of preverbal communication (i.e., joint attention) and later language (Schertz, Odom, et al., 2018; Trevarthen \& Hubley, 1978). It may also help children share in the perspectives of others (Harrist \& Waugh, 2002). For many young children with autism, however, engaging in dyadic turn-taking exchanges may be challenging, particularly for social purposes (Chiang, Soon, Lin, \& Rogers, 2008; Clifford \& Dissanayake, 2009). Chiang and colleagues (2008), for instance, found that, when compared to children with typical development, children with autism showed fewer instances of turn taking when it centered around actions with another person (e.g., teasing, or tickling games). Because of this potential challenge in turn taking, children with autism may have difficulty learning it on their own and may require support in this form of preverbal social communication. Given its importance in childhood development, turn taking has been incorporated as a component of multiple interventions for children with autism, such as Floortime (Greenspan \& Wieder, 2006), Joint Attention Mediated Learning (Schertz, Odom, et al., 2018), and behavioral approaches (e.g., Isaksen \& Holth, 2009). In a study by Isaksen and Holth (2009), for instance, children received intensive behavioral training in turn-taking use. Schertz, Odom, and colleagues (2018) also incorporated a turn-taking component in intervention to promote joint attention learning in toddlers. In addition to being a component of intervention, turn taking is a defined task on the Early Social Communication Scales, which is used to measure children's use of preverbal communication and helps in identifying potential developmental delays (Mundy et al., 2003), further indicating the importance placed on this form of preverbal communication in childhood development.

\section{Distinguishing Turn Taking by Function}

Turn taking at its broadest definition is a simple, back-and-forth communicative exchange between partners (Lee \& Schertz, 2020); however, it may also serve varying functions (i.e., social, or instrumental) depending on the intent behind the interaction (e.g., Alpert \& Kaiser, 1992; Greenspan \& Wieder, 2006; Isaksen \& Holth, 2009; Schertz, Odom, et al., 2018). The social function has been defined as a sharing of attention through common interests with another person, such as can be observed during joint attention overtures, or while commenting (Cochet \& Byrne, 2016; Schertz, Call-Cummings, Horn, Quest, \& Steffen Law, 2018). The social function may be observed, for instance, when a child engages in block building with a parent, during which time the child shows playful intent by smiling and clapping their hands with excitement. In this example, the child is displaying signs of active, meaningful engagement with the parent. The instrumental function, on the other hand, is marked by requesting or following the request of a communicative partner and serves the purpose of achieving a goal for the purpose of the self, rather than the partner or for mutual interest sharing (Cochet \& Byrne, 2016; Schertz, Call-Cummings, et al., 2018). The instrumental function may be observed, for example, when a child points to request a desired toy from a parent and the parent responds to that request by handing the toy to the child. In this scenario, the child is 
seeking to obtain the toy for their own purposes and is not pointing to share interest or to engage socially with the parent.

Although the instrumental and social communicative functions are distinguishable, there is limited research exploring the differences in turn-taking functions. Other forms of preverbal communication, such as eye gaze and pointing, have been differentiated by social and instrumental function (e.g., Cochet \& Byrne, 2016; Mundy et al., 1986). Cochet and Byrne (2016), for instance, analyzed the differential relationship of social vs. instrumental pointing on later language and symbolic gesture use and found that the social function was related to later development, but the instrumental function was not. Determining the function behind a turn-taking exchange in intervention may be especially relevant for children with autism, who have a core challenge in social communication and who may also have less difficulty with and who may favor instrumental over social communication (Adamson, McArthur, Markov, Dunbar, \& Bakeman, 2001; Klin, Lin, Gorrindo, Ramsay, \& Jones, 2009; Mundy, 1995; Sigman \& Ruskin, 1999). While not all interventions for children with autism employ the same strategies or follow the same approach and design, when the goal of an intervention is to support social communication outcomes through a turntaking component, how turn taking is being used by the child and their partner (i.e., its function), should be carefully considered.

Even though turn taking is well established as a component of intervention and is regarded as having important developmental implications, there remains general inconsistency among the current intervention literature in how turn taking is defined based on function, with some interventions defining turn taking socially (e.g., Greenspan \& Wieder, 2006; Schertz, Odom, et al., 2018) and some defining it instrumentally (e.g., Isaksen \& Holth, 2009; Koegel et al., 1989), warranting a need for further, more exhaustive review of how turn taking is operationally defined by function in the current autism intervention research. Prior to the present study, there have not been any such reported explorations of current literature. Therefore, the purpose of this review is to determine how turn taking is operationally defined based on communicative function in studies on interventions for young children with autism. Similar review methodologies have revealed key variations across study terminology that ultimately affect study transparency and replicability for future research and practice (Kamenopoulou, Ali, \& Ockelford, 2021). A secondary purpose is to explore other intervention content, including participants, autism assessment data, settings, agents, design, strategies, and target outcomes to provide context to how turn taking is promoted in each identified intervention study.

\section{Method}

The authors searched the online databases PsychINFO and Academic Search Premier, two of the foremost databases in the social sciences, to identify relevant peer-reviewed journal articles published in English. Additionally, online first articles were searched on the following journal sites: Autism, Focus on Autism and Other Developmental Disabilities, Journal of Autism and Developmental Disorders, Journal of Early Intervention, Research in Autism Spectrum Disorders, and Topics in Early Childhood Special Education. Dates of publication were limited to the 10-year span between 2010 and 2020 to ensure that the most recent research was included in the review, particularly studies that define autism under the current diagnostic criteria published in the Diagnostic and Statistical Manual of Mental Disorders-V (APA, 2013). Specific keywords used in identifying potentially relevant studies include autism or ASD and intervention and (turn taking or reciprocal or dyadic). The terms "reciprocal" and "dyadic" were searched since not all researchers use the same terminology to describe back-and-forth exchanges. This initial search yielded 439 potentially relevant studies.

\section{Inclusion \& Exclusion Criteria}

After an initial search of the chosen keywords filtered by the initial inclusion parameters, the first and second authors independently screened articles for adherence to additional inclusion criteria. To be included in the current review, identified literature must meet the following criteria: a) be an intervention study involving young children diagnosed with or at risk for autism between the ages of 0 to 8 (following 
the definition of early childhood provided by the Division for Early Childhood of the Council for Exceptional Children [DEC] 2014); b) include an embedded preverbal turn-taking component; c) describe turn taking between two communicative partners and not between a child and a non-human entity (e.g., a robot or a tablet application); and d) present turn taking in the methods section as an operational definition, such as in study coding criteria, measures, or the intervention description, and not in the introduction or literature review. For the present study, a turn-taking component is defined as any use of turn taking in the intervention for the promotion of targeted outcomes. For example, in the study by Schertz, Odom, and colleagues (2018), turn taking was promoted in conjunction with other components in intervention to help children build their competency towards joint attention.

Of the 439 originally identified studies, most were excluded because they were not intervention studies (e.g., literature reviews or essays), did not have participants with autism, or had participants that were older than 8 years of age $(n=359)$. To maintain consistency with inclusion criteria, studies with children older than 8 years of age were excluded, even if some of the children were under 8 . Fourteen studies were excluded because children engaged exclusively with a non-human entity during intervention; however, one robot-involved study had a human turn-taking condition and was included (David, Costescu, Matu, Szentagotai, \& Dobrean, 2020). If a study was a replication of another identified study, only the most current study was included; however, if the replication study referred to the original study regarding specific intervention procedures and definitions, the definition of the original study was considered for this literature review. Studies included in this review were not limited by research design if the intervention had a turn-taking component. It is important to note that turn taking may be defined as verbal communicative acts that involve children initiating or responding to turns verbally (McFadden, Kamps, \& Heitzman-Powell, 2014); however, the present study focuses on preverbal turn taking. Therefore, interventions that only used a verbal turn-taking component to promote later outcomes were excluded from the review. Studies that incorporated both preverbal and verbal turn taking were included, however. Numerous studies $(n=74)$ were excluded because only a verbal turn-taking component was defined and/or preverbal turn taking was not an embedded intervention component. After independent screening procedures were conducted, the authors met and conducted additional screening for duplication, and agreed that a total of 14 studies met the inclusion criteria for this review.

\section{Coding}

The first and second authors independently reviewed and coded each of the 14 articles based on the following established coding criteria. We then met virtually to discuss our findings and address differences in coding until agreement was met, a coding method commonly reported in rigorous literature reviews (e.g., Cooper, 2010; Pennington \& Delano, 2012; Schertz, Reichow, Tan, Vaiouli, \& Yildirim, 2012). To code turn taking by function, the authors followed the definitions of social and instrumental communication as described by Lee and Schertz (2020) and Schertz, Call-Cummings, and colleagues (2018). Studies with operational definitions describing back-and-forth exchanges for the purpose of sharing interest, such as in an object or activity, and engaging socially with a communicative partner were coded as "Social" (Lee \& Schertz, 2020; Schertz, Call-Cummings, et al., 2018). Indications of the "Social" function may include positive affect (e.g., smiling, or facial excitement), child-led interactions, and a lack of agent instructions or requests (Lee \& Schertz, 2020; Schertz, Call-Cummings, et al., 2018). Studies with operational definitions describing back-and-forth exchanges for task-oriented purposes, such as by following or initiating a direct instruction or request, or to acquire something without consideration of the communicative partner's interests were coded as "Instrumental" (Schertz, Call-Cummings, et al., 2018). The "Instrumental" function may be identified by a focus on completing a task, such as taking turns as part of following rules in a board game or responding to an agent's directions (e.g., "Your turn" or "My turn") (Schertz, Call-Cummings, et al., 2018).

For the current study, a third coding criterion was applied. Studies in which the turn-taking function was unclear were coded as "Undetermined." The turn-taking function was unclear if the study: (1) described a back-and-forth exchange without indicating if the purpose was for instrumental or social 
purposes or (2) the term "turn taking" was used, but the form (i.e., the back-and-forth nature) was unclear. Additional intervention features, including participants, autism assessment data, settings, agents, design, approach, and target outcomes, were explored in the literature to give fuller context to each intervention study.

\section{Results}

The findings from the 14 studies coded for the present literature review are presented in (Table 1).

Table 1. Intervention content and turn-taking functions

\begin{tabular}{|c|c|c|c|c|c|c|c|c|}
\hline \multirow[b]{2}{*}{ Study } & \multicolumn{7}{|c|}{ Intervention features } & \multirow{2}{*}{$\begin{array}{l}\text { Turn-taking } \\
\text { Function }\end{array}$} \\
\hline & $\begin{array}{c}\text { Participants } \\
n \text { (age)/M:F }\end{array}$ & $\begin{array}{c}\text { Setting } \\
\mathrm{s}\end{array}$ & $\begin{array}{l}\text { Assessm } \\
\text { ents }\end{array}$ & Agents & Design & Approach & Target outcomes & \\
\hline $\begin{array}{l}\text { David et } \\
\text { al. (2020) }\end{array}$ & $\begin{array}{c}5(3-5 \\
\text { years }) / 3: 2\end{array}$ & Clinic & $\begin{array}{c}\text { ADOS } \\
7.2(1.7)\end{array}$ & Therapist & $\begin{array}{c}\text { Multiple } \\
\text { baseline } \\
\text { alternating } \\
\text { treatments }\end{array}$ & Behavioral & $\begin{array}{c}\text { Turn taking } \\
\text { (Undetermined); } \\
\text { Preverbal social } \\
\text { communication } \\
\text { (e.g., positive } \\
\text { affect, } \\
\text { engagement, eye } \\
\text { contact); } \\
\text { Changes in some } \\
\text { behaviors }\end{array}$ & Undetermined \\
\hline $\begin{array}{c}\text { Dawson } \\
\text { et al. } \\
(2010)\end{array}$ & $\begin{array}{c}24(18-30 \\
\text { months }) / 3.5: \\
1^{a}\end{array}$ & Home & $\begin{array}{c}\text { ADOS } \\
7.2(1.7)\end{array}$ & $\begin{array}{l}\text { Parents } \\
\text { and } \\
\text { therapist } \\
\text { s }\end{array}$ & $\mathrm{RCT}$ & Combined & $\begin{array}{c}\text { Reduced } \\
\text { symptom } \\
\text { severity } \\
\text { (including } \\
\text { improved } \\
\text { overall social } \\
\text { communication); } \\
\text { Changes in } \\
\text { cognitive \& } \\
\text { adaptive } \\
\text { behavior; } \\
\text { Language }\end{array}$ & Social \\
\hline $\begin{array}{c}\text { Gengoux } \\
\text { et al. } \\
(2019)\end{array}$ & $\begin{array}{c}22(2-6 \\
\text { years }) / 18: 4\end{array}$ & Clinic & $\begin{array}{c}\text { ADOS } \\
7.7(1.4)\end{array}$ & $\begin{array}{l}\text { Parents } \\
\text { and } \\
\text { therapist } \\
\text { s }\end{array}$ & $\begin{array}{c}\text { Uncontroll } \\
\text { ed trial }\end{array}$ & $\begin{array}{l}\text { Developm } \\
\text { ental }\end{array}$ & $\begin{array}{c}\text { Reduced } \\
\text { symptom } \\
\text { severity } \\
\text { (including } \\
\text { improved } \\
\text { overall social } \\
\text { communication, } \\
\text { \& changes in } \\
\text { RRBs); Language }\end{array}$ & Social \\
\hline $\begin{array}{l}\text { Green et } \\
\text { al. (2017) }\end{array}$ & $\begin{array}{c}28(7-10 \\
\text { months }) / 12 \\
5: 1^{\mathrm{a}}\end{array}$ & Home & $\begin{array}{c}\text { AOSI } \\
10.04 \\
(4.6)\end{array}$ & Parents & RCT & $\begin{array}{c}\text { Developm } \\
\text { ental }\end{array}$ & $\begin{array}{l}\text { Turn taking } \\
\text { (Social); } \\
\text { Reduced } \\
\text { symptom } \\
\text { severity } \\
\text { (including } \\
\text { improved } \\
\text { overall social } \\
\text { communication, } \\
\text { \& changes in } \\
\text { RRBs) }\end{array}$ & Social \\
\hline
\end{tabular}


Kwangwon LEE \& Ashley STAGGS

\begin{tabular}{|c|c|c|c|c|c|c|c|c|}
\hline \multirow[b]{2}{*}{ Study } & \multicolumn{7}{|c|}{ Intervention features } & \multirow{2}{*}{$\begin{array}{l}\text { Turn-taking } \\
\text { Function }\end{array}$} \\
\hline & $\begin{array}{l}\text { Participants } \\
n \text { (age)/M:F }\end{array}$ & $\begin{array}{c}\text { Setting } \\
\mathrm{s}\end{array}$ & $\begin{array}{c}\text { Assessm } \\
\text { ents }\end{array}$ & Agents & Design & Approach & Target outcomes & \\
\hline $\begin{array}{c}\text { Kemp, } \\
\text { Stephenso } \\
\text { n, } \\
\text { Cooper, \& } \\
\text { Hodge } \\
(2019)\end{array}$ & $\begin{array}{c}3 \text { (all under } \\
\text { the age of } \\
5) / 3: 0\end{array}$ & $\begin{array}{l}\text { Childca } \\
\text { re } \\
\text { centers }\end{array}$ & N/A & Peers & $\begin{array}{l}\text { Multiple } \\
\text { probe } \\
\text { across } \\
\text { participan } \\
\text { ts }\end{array}$ & Behavioral & $\begin{array}{c}\text { Turn taking } \\
\text { (Instrumental) }\end{array}$ & Instrumental \\
\hline $\begin{array}{l}\text { Kim \& } \\
\text { Clarke } \\
(2015)\end{array}$ & $\begin{array}{c}2(4 \\
\text { years }) / 2: 0\end{array}$ & Home & N/A & $\begin{array}{l}\text { Research } \\
\text { er }\end{array}$ & $\begin{array}{l}\text { Multiple } \\
\text { baseline } \\
\text { across } \\
\text { participan } \\
\text { ts }\end{array}$ & Behavioral & $\begin{array}{l}\text { Turn taking } \\
\text { (Instrumental) }\end{array}$ & Instrumental \\
\hline $\begin{array}{c}\text { Raulston, } \\
\text { Hansen, } \\
\text { Frantz, } \\
\text { Machalice } \\
\text { k, \& } \\
\text { Bhana } \\
(2020)\end{array}$ & $\begin{array}{c}3(3-6 \\
\text { years)/2:1 }\end{array}$ & Home & $\begin{array}{l}\text { CARS-2 } \\
35(4.44)\end{array}$ & $\begin{array}{l}\text { Parents } \\
\text { and } \\
\text { peers }\end{array}$ & $\begin{array}{l}\text { Multiple } \\
\text { probe } \\
\text { across } \\
\text { participan } \\
\text { ts }\end{array}$ & Behavioral & $\begin{array}{l}\text { Verbal \& } \\
\text { nonverbal } \\
\text { communication } \\
\text { (e.g., mands \& } \\
\text { response to } \\
\text { mands) }\end{array}$ & Instrumental \\
\hline $\begin{array}{l}\text { Rieth et } \\
\text { al. (2014) }\end{array}$ & $\begin{array}{c}6 \text { (ages } 2 \text { to } \\
4) / 4: 2\end{array}$ & $\begin{array}{l}\text { Clinic/ } \\
\text { Home }\end{array}$ & $\begin{array}{c}\text { ADOS } \\
2 \text { ASD \& } \\
4 \\
\text { Autismb }^{b}\end{array}$ & $\begin{array}{c}\text { Therapist } \\
\text { s }\end{array}$ & $\begin{array}{l}\text { Multiple } \\
\text { baseline } \\
\text { alternating } \\
\text { treatments }\end{array}$ & Behavioral & $\begin{array}{c}\text { Requesting; } \\
\text { Commenting; } \\
\text { Play }\end{array}$ & Instrumental \\
\hline $\begin{array}{c}\text { Rollins, } \\
\text { Campbell, } \\
\text { Thibodea } \\
\text { u } \\
\text { Hoffman, } \\
\text { \& Self } \\
(2016)\end{array}$ & $\begin{array}{l}4 \text { (All under } \\
36 \\
\text { months)/4:0 }\end{array}$ & Home & $\begin{array}{c}\text { CARS-2 } \\
44.62 \\
(3.54)\end{array}$ & Parents & $\begin{array}{l}\text { Multiple } \\
\text { baseline } \\
\text { across } \\
\text { participan } \\
\text { ts }\end{array}$ & Combined & $\begin{array}{l}\text { Turn taking } \\
\text { (Undetermined); } \\
\text { Eye contact; } \\
\text { Social } \\
\text { engagement; } \\
\text { Verbal turn } \\
\text { taking }\end{array}$ & Undetermined \\
\hline $\begin{array}{l}\text { Schertz, } \\
\text { Odom, et } \\
\text { al. (2018) }\end{array}$ & $\begin{array}{c}73 \text { (ages 16- } \\
30 \text { months)/ } \\
58: 15^{\mathrm{a}}\end{array}$ & Home & $\begin{array}{c}\text { ADOS-T } \\
16.36 \\
(3.45)\end{array}$ & Parents & $\mathrm{RCT}$ & $\begin{array}{l}\text { Developm } \\
\text { ental }\end{array}$ & $\begin{array}{l}\text { Preverbal social } \\
\text { communication } \\
\text { (i.e., facial } \\
\text { focusing, turn } \\
\text { taking, \& joint } \\
\text { attention) }\end{array}$ & Social \\
\hline $\begin{array}{l}\text { Shire, } \\
\text { Shih, } \\
\text { Bracaglia, } \\
\text { Kodjoe, \& } \\
\text { Kasari } \\
(2020)\end{array}$ & $\begin{array}{c}50(\text { mean } \\
\text { age } 32.42 \\
\text { months)/40: } \\
10\end{array}$ & $\begin{array}{l}\text { Childca } \\
\text { re } \\
\text { centers }\end{array}$ & N/A & $\begin{array}{c}\text { Paraprof } \\
\text { essionals } \\
\text { and } \\
\text { peers }\end{array}$ & $\mathrm{RCT}$ & Combined & $\begin{array}{c}\text { Preverbal social } \\
\text { communication } \\
\text { (e.g., joint } \\
\text { attention, joint } \\
\text { engagement); } \\
\text { Play; Language }\end{array}$ & Instrumental \\
\hline $\begin{array}{c}\text { Therrien } \\
\text { \& Light } \\
\text { (2018) }\end{array}$ & $\begin{array}{c}5(3 \text { to } 6 \\
\text { years)/5:0 }\end{array}$ & $\begin{array}{l}\text { Childca } \\
\text { re } \\
\text { centers }\end{array}$ & $\begin{array}{c}\text { CARS-2 } \\
34.9 \\
(5.85)\end{array}$ & $\begin{array}{c}\text { Peers } \\
\text { and } \\
\text { intervent } \\
\text { ionist }\end{array}$ & $\begin{array}{l}\text { Multiple } \\
\text { probe } \\
\text { across } \\
\text { participan } \\
\mathrm{t}\end{array}$ & Behavioral & $\begin{array}{c}\text { Turn taking } \\
\text { (Instrumental); } \\
\text { Preverbal social } \\
\text { communication } \\
\text { (e.g., joint } \\
\text { engagement) }\end{array}$ & Instrumental \\
\hline $\begin{array}{l}\text { Thompso } \\
\text { n, } \\
\text { McFerran, } \\
\text { \& Gold } \\
(2014)\end{array}$ & $\begin{array}{l}12 \text { (mean } \\
\text { age } \\
43.92) / 8: 4\end{array}$ & Home & $\begin{array}{c}\text { SRS-PS } \\
87.42 \\
(15.84)\end{array}$ & $\begin{array}{c}\text { Parents } \\
\text { and } \\
\text { therapist } \\
\text { s }\end{array}$ & $\mathrm{RCT}$ & $\begin{array}{l}\text { Developm } \\
\text { ental }\end{array}$ & $\begin{array}{l}\text { Social } \\
\text { interactions \& } \\
\text { responsivity; } \\
\text { Improved } \\
\text { parent-child }\end{array}$ & Undetermined \\
\hline
\end{tabular}


Defining turn taking in intervention...

\begin{tabular}{|c|c|c|c|c|c|c|c|c|}
\hline \multirow[b]{2}{*}{ Study } & \multicolumn{7}{|c|}{ Intervention features } & \multirow{2}{*}{$\begin{array}{l}\text { Turn-taking } \\
\text { Function }\end{array}$} \\
\hline & $\begin{array}{c}\text { Participants } \\
n \text { (age)/M:F }\end{array}$ & $\begin{array}{c}\text { Setting } \\
\mathrm{s}\end{array}$ & $\begin{array}{l}\text { Assessm } \\
\text { ents }\end{array}$ & Agents & Design & Approach & Target outcomes & \\
\hline \multirow[b]{2}{*}{$\begin{array}{l}\text { Wang } \\
\text { (2017) }\end{array}$} & \multirow[b]{2}{*}{$\begin{array}{c}4(2-4 \\
\text { years }) / 3: 1\end{array}$} & \multirow[b]{2}{*}{ Home } & \multirow[b]{2}{*}{ N/A } & \multirow[b]{2}{*}{ Parents } & & & $\begin{array}{l}\text { relationships; } \\
\text { Language }\end{array}$ & \multirow[b]{2}{*}{ Undetermined } \\
\hline & & & & & $\begin{array}{l}\text { Multiple } \\
\text { baseline } \\
\text { across } \\
\text { participan } \\
\text { ts }\end{array}$ & Behavioral & $\begin{array}{l}\text { Turn taking } \\
\text { (Undetermined); } \\
\text { Decreased } \\
\text { refusal behaviors }\end{array}$ & \\
\hline
\end{tabular}

Note: RCT = Randomized controlled trial; RRB = Restrictive and Repetitive Behaviors; Combined = contains aspects of both behavioral and developmental approaches; ADOS = Autism Diagnostic Observation Schedule; ADOS-T = Autism Diagnostic Observation Schedule-Toddler; AOSI = Autism Observation Schedule for Infants; CARS-2 = Childhood Autism Rating Scale; SRS-PS = Social Responsiveness Scale-Preschool; N/A = Assessment data not provided.

aApproximate ratio, actual numbers not reported

bOnly ADOS classification available

\section{Intervention Features}

\section{Participants \& Autism Assessment Data}

Among the 14 studies reviewed, children ranged in age from 7 months to 6 years at the start of intervention. Most child participants ( $n \sim 195)$ were male, but exact figures could not be determined across studies since not all articles reported the exact participant numbers (i.e., Dawson et al., 2010; Green et al., 2017; Schertz, Odom, et al., 2018). While all studies included children in the early childhood stage of development (up to 8 years old), only eight of the studies would be classified as early interventions (i.e., included children under the age of three) under Part $C$ of the Individuals with Disabilities Education Improvement Act (2004), a United States special education law that mandates rights and protections for children with disabilities. All studies indicated that child participants had or were at high risk for a diagnosis of autism spectrum disorder as determined by one or more of the following tools: the Autism Diagnostic Observation Schedule (ADOS) (Lord et al., 1999), the Autism Diagnostic Observation ScheduleToddler (ADOS-T) (Lord, Luyster, Gotham, \& Guthrie, 2012), the Autism Observation Schedule for Infants (AOSI) (Bryson, Zwaigenbaum, McDermott, Rombough, \& Brian, 2008), the Childhood Autism Rating Scale, Second Edition (CARS-2) (Schopler, Van Bourgondien, Wellman, \& Love, 2010); the Social Responsiveness Scale-Preschool (SRS-PS) (Constantino \& Gruber, 2005), and clinical expertise (i.e., Kemp et al., 2019; Kim \& Clarke, 2015; Shire et al., 2020; Wang, 2017).

\section{Agents \& Settings}

Most of the interventions were implemented by parents to some degree (i.e., as the sole agent or as a co-agent) $(\mathrm{n}=8)$. Peers as intervention agents were involved in four of the studies as sole agent or coagent. Five studies incorporated therapists as agents. One study was partially implemented by classroom paraprofessionals (Shire et al., 2020), one study was partially implemented by an interventionist (Therrien \& Light, 2018), and another study was conducted by the researcher (Kim \& Clarke, 2015). The agents of one intervention included therapists and a robot (David et al., 2020); however, for the purpose of the current review, only the human therapists were considered intervention agents. Most interventions took place in participants' homes $(n=9)$. Of those, one study was conducted in the home setting and the clinic setting (Rieth et al., 2014). Two interventions were conducted exclusively in clinical settings, which included therapy clinics and autism treatment centers (David et al., 2020; Gengoux et al., 2019). Three interventions were conducted in childcare centers, which included early childhood classrooms and day care settings (Kemp et al., 2019; Shire et al., 2020; Therrien \& Light, 2018).

\section{Design}

Most of the studies in this review $(n=8)$ used single subject designs to test interventions. Of these, three reported the use of a multiple baseline across participants design (i.e., Kim \& Clarke, 2015; Rollins et 
al., 2016; Wang, 2017), three used a multiple probe across participants design (i.e., Kemp et al., 2019; Raulston et al., 2020; Therrien \& Light, 2018), and two used an alternating treatments design (i.e., David et al., 2020; Rieth et al., 2014). Five intervention studies were randomized controlled trials (i.e., Dawson et al., 2010; Green et al., 2017; Schertz, Odom, et al., 2018 Shire et al., 2020; Thompson et al., 2014), and one study was an uncontrolled trial (i.e., Gengoux et al., 2019).

\section{Approach}

The majority of studies reviewed $(n=7)$ implemented behavioral approaches to intervention, which follow the principles of applied behavior analysis (i.e., David et al., 2020; Kemp et al., 2019; Kim \& Clarke, 2015; Raulston et al., 2020; Rieth et al., 2014; Therrien \& Light, 2018; Wang, 2017). Of these, two studies employed prompting and reinforcement to elicit turn taking from participants (i.e., Kim \& Clarke, 2015; Therrien \& Light, 2018). David and colleagues (2020) implemented Discrete Trial Training, a technique that breaks down behaviors into smaller components, to teach turn taking to children with autism. In Raulston and colleagues' study (2020), parents used a coaching model to encourage turn taking in their children during game play (e.g., while pretend fishing or in proprietary games that require give and take) while at playdates with peers. Rieth and colleagues (2014) used Pivotal Response Training to increase use of turn taking, communication, and play through a turn-taking component. In one study, peers with typical development were trained on how to teach children with autism to engage in turn taking through a peer mediated intervention (Kemp et al., 2019). Wang (2017) utilized video modeling supplemented by book reading in intervention to demonstrate turn taking to parents and their children with autism.

Developmental approaches follow the principles of developmental science, are designed to follow the developmental trajectories of young children, and emphasize the importance of child-centered approaches to learning (Rogers \& Wallace, 2011). Of the studies reviewed, four followed a developmental approach. Specifically, developmental parent mediation was solely implemented in two studies (Green et al., 2017; Schertz, Odom, et al., 2018). In Schertz, Odom, et al. (2018), parents were supported with the guidance of an interventionist on how to promote turn taking in their young children in daily routines. Green and colleagues (2017) used a video program to guide parents in promoting social communication, including turn taking, in their young children. In conjunction with parent mediation, therapist modeling was used in one study to demonstrate intervention procedures to parents, who then implemented what they observed with their children (Gengoux et al., 2019). Only one study reported the use of music therapy that followed a developmental approach to intervention. Specifically, Thompson and colleagues (2014) promoted turn taking through family-centered music therapy sessions which alternated between highly structured sing along sessions to less structured activities that involved playing music to elicit turn taking.

Combined approaches, also known as comprehensive program models, integrate aspects of developmental and behavioral approaches in intervention (Odom, Boyd, Hall, \& Hume, 2010). A combined approach was used in three studies. One study combined behavioral intervention with parent training under a comprehensive program model called the Early Start Denver Model (ESDM) (Dawson et al., 2010). Rollins et al. (2016) combined parent mediation and an early intensive behavioral intervention. Finally, children engaged with peers as one condition of intervention and with paraprofessionals in another condition in a classroom-based setting in one comprehensive program model (Shire et al., 2020).

\section{Target Outcomes}

Interventions that included turn taking varied in their targeted outcomes. Most interventions $(n=9)$ targeted improvements in preverbal forms of social communication, such as eye gaze, joint engagement, joint attention, and turn taking, all of which serve the purpose of sharing interest with a communicative partner and engaging socially (i.e., David et al., 2020; Dawson et al., 2010; Gengoux et al., 2019; Green et al., 2017; Rollins et al., 2016; Schertz, Odom, et al., 2018). One study sought to improve verbal commenting, which also serves a purpose of sharing interest with another person (Rieth et al., 2014). Through three of the interventions, researchers targeted increased instrumental turn-taking frequency (i.e., Kemp et al., 2019; Kim \& Clarke, 2015; Therrien \& Light, 2018). Instrumental verbal and preverbal forms of communication, including requesting, and responses to and initiations of mands were sought in two studies (Raulston et 
al., 2020; Reith et al., 2014). Turn taking with an "Undetermined" function as an outcome was sought in three studies (i.e., David et al., 2020; Rollins et al., 2016; Wang, 2017).

Two interventions targeted play competencies, such as pre-symbolic play and cooperative block building (Rieth et al., 2014; Shire et al., 2020). Improvements in expressive and receptive language were targeted in four studies (i.e., Dawson et al., 2010; Gengoux et al., 2019; Shire et al., 2020; Thompson et al., 2014). Five interventions addressed behavioral outcomes as well, such as avoidance and aggression, and outcomes associated with restrictive and repetitive behaviors, another core challenge for individuals with autism (i.e., David et al., 2020; Dawson et al., 2010; Gengoux et al., 2019; Green et al., 2017; Wang, 2017). Wang (2017), for example, used video modeling not only to promote turn taking in children with autism, but to also decrease refusal behaviors. Lastly, Dawson and colleagues (2010), in addition to their other targeted outcomes, sought improvements in cognition.

\section{Turn-Taking Function}

Social. Of the 14 studies reviewed, four defined turn taking as having a social function. Schertz, Odom, and colleagues (2018) defined turn taking as synchronous, back-and-forth engagement between a child and their parent for the purpose of sharing social interest. Turn taking in this study was child led and parents encouraged reciprocal engagement by following the child's interests and using positive affect (e.g., smiling, excited facial expressions, and praise), rather than directly instructing children in what to do (Schertz, Odom, et al., 2018). Similarly, Gengoux and colleagues (2019) define reciprocal turn taking as being child led and based on child interests, is supported by use of positive affect and praise, and as occurring in natural play settings. Dawson et al. (2010) implemented the ESDM in their replication study and the definition of turn taking was not directly quoted in the article; however, the ESDM defines it as having a social function, in which turn taking is not prompted or requested, but is natural, synchronous, and often play-based with children (Rogers et al., 2012). Finally, Green et al. (2017) defines turn taking, which they term "dyadic interaction," as back-and-forth synchronous engagement, that is nondirective (i.e., unprompted and occurring naturally), and is child led.

Instrumental. Most of the reviewed studies $(n=6)$ defined turn taking as having an instrumental function. Kemp and colleagues (2019) defined turn taking as a back-and-forth exchange involving opening and closing graphics of animals in a tablet application; therefore, the goal of the exchange was to accomplish a specific task (i.e., using the application) rather than sharing interest with a communicative partner. Additionally, children in this study could be verbally instructed to take turns by peers. Similarly, Kim and Clarke (2015) utilized a tablet to encourage turn taking with different toys in their participants; however, children were not allowed to touch the tablet and it was only used for prompting purposes. Like Kemp et al. (2019) and Kim and Clarke (2015), Raulston and colleagues (2020) defined turn taking as part of engaging with toys or taking turns in a game with the main purpose of completing a task. In Therrien and Light (2018), interventionists implemented continuous prompting (e.g., "Your turn") and modeling to teach children how to initiate and respond to turn taking with their peers, the focus being on accomplishing the back-and-forth exchange rather than on social engagement. Rieth and colleagues (2014) also used modeling and contingency to teach turn taking to children, such as by requesting a turn from the child and then asking the child to respond with a turn, rather than having the children initiate and respond for their own interest and volition. Finally, Shire et al. (2020) used modeling, prompting, and fading to teach children to take turns with the help of their peers. The authors use the following example to illustrate what their turn-taking component may look like, "if a peer were to hand a block to the child and the child did not notice, the (paraprofessional) may point out the peer's initiation and provide environmental, verbal, or physical support for the child to receive the block from the peer" (Shire et al., 2020, p. 2145). In this example, the child is engaging in a back-and-forth exchange, but for the purpose of achieving a task-oriented goal of taking a turn rather than for engaging socially and sharing interest with a peer.

Undetermined. Turn taking was coded as "Undetermined" in four studies because the function of turn taking could not be determined based on the operational definition provided. Wang's (2017) study, which was further categorized as "function unclear," defined turn taking as "a pair of one initiation, either 
verbal or non-verbal interaction, and one response, either verbal or non-verbal interaction" (p. 7). This operational definition of turn taking is defined as back-and-forth engagement, but the purpose behind the interaction is unclear.

Three of the four undetermined studies were categorized as both "function unclear" and "form unclear" (i.e., David et al., 2020; Rollins et al., 2016; Thompson et al., 2014). Rollins and colleagues (2016) defined their nonverbal turn-taking coding criteria as any instance "when the child took a turn with an object following the partner's initiation" (p. 224). From this definition, the function, or communicative partners' intent, could not be determined and the child's initiation, which completes the reciprocal exchange, was not included. Thompson and colleagues (2014) defined turn taking in a music therapy context, in which turn taking was defined as instances when "the music therapist would play a predictable harmonic structure that ended with an unresolved cadence, and then pause to wait for the child to respond" (p. 844). Again, the function behind the exchange, or why the child is responding to this initiation, is not clear, and the child's initiation is not defined. Similarly, turn taking as defined by David et al. (2020) was categorized as "Undetermined" because their definition only considers the child's response to the partner's initiation. Turn taking in this study was primarily defined as "the performance of the child to wait his or her turn" (David et al., 2020, p. 34). From this definition, neither the form nor the function is clear.

\section{Conclusion and Discussion}

Previous studies have explored the differential effects and relationships of instrumental vs. social preverbal communication on later developmental outcomes, such as language and joint attention, and identified differences in each function, thus indicating the importance of considering communicative intent (e.g., Cochet \& Byrne, 2016; Mundy et al., 1986). The present literature review focused on turn taking, a form of preverbal communication that is incorporated in intervention for children with autism, and how it is operationally defined based on communicative function across studies. The results of this review indicate that there is variability in how turn taking is defined in the current literature on interventions for young children with autism.

Specifically, most of the studies provided clear definitions of instrumental turn taking. Social turn taking was also clearly defined in four of the reviewed studies. However, four of the studies included in this review defined turn taking in ways that left the function undetermined. For instance, the author of one study defined the form of turn taking (i.e., a back-and-forth exchange), indicating that turn taking required both an initiation and response from the child, but the function, or the intent behind the interaction, was not clear (Wang, 2017). Additionally, although the term "turn taking" was used in David et al. (2020), Rollins et al. (2016), and Thompson et al. (2014), their definitions only consider the child's response and not their initiation. Turn taking involves a level of give and take from both communicative partners and may be a form of dyadic synchrony that promotes children's awareness that their partners have their own thoughts and interests, and, through intersubjectivity, children may share in their partner's interests (Harrist \& Waugh, 2002). Therefore, consideration of each partner's response, as well as their initiations, should be accounted for when defining and including turn taking in an intervention for children with autism, who may have inherent difficulty in turn-taking exchanges, particularly for social purposes (Chiang et al., 2008; Clifford \& Dissanayake, 2009). These findings indicate that greater clarity and specificity is needed when defining turn taking not only by function, but also by form in intervention research. Clarifying turn taking by form (i.e., the back-and-forth exchange) can be accomplished by defining how children initiate turn taking with a partner and how they respond to a partner's turn-taking bid. To clarify turn taking by function, researchers should detail if the child and their communicative partner are engaging in a turn-taking routine for social or instrumental purposes based on the coding criteria presented in this article and by other researchers who have studied communicative intent (e.g., Cochet \& Byrne, 2016; Schertz, Call-Cummings, et al., 2018). Additionally, among the articles on intervention for young children with autism published within the last decade, only 14 incorporated a preverbal turn-taking component defined as an exchange between two human communicative partners. Of these, only four defined turn taking socially. Given the developmental implications of turn taking on 
later outcomes, especially those related to social communication (e.g., joint attention) (Schertz, Odom, et al., 2018), socially defined turn taking should be included more often as a component of interventions for children with autism in future research.

Among the studies reviewed, the intervention approach seems to correspond with how turn taking is defined by function. Five of the 6 interventions that defined turn taking instrumentally followed a behavioral approach to intervention (i.e., Kemp et al., 2019; Kim \& Clarke, 2015; Raulston et al., 2020; Rieth et al., 2014; Therrien \& Light, 2018). Three of the 4 interventions that defined turn taking socially followed a developmental approach to intervention (i.e., Gengoux et al., 2019; Green et al., 2017; Schertz, Odom, et al., 2018). These findings are not unanticipated given the nature of each intervention approach. Behavioral interventions are primarily designed to increase an observable skill and/or decrease some behaviors (Rogers \& Wallace, 2011), which aligns well with the task-oriented nature of the instrumental function (Schertz, Call-Cummings, et al., 2018). Developmental approaches, on the other hand, focus on how children develop naturally and have an especial importance in helping them to gain competency in preverbal social communication, such as social turn taking (Wetherby \& Woods, 2008). The interventions that followed combined approaches varied in how turn taking was defined, with one defining it socially (i.e., Dawson et al., 2010), one defining it instrumentally (i.e., Shire et al., 2020), and one defining turn taking with an undetermined function (i.e., Rollins et al., 2016). This variation among the combined approaches to intervention in how turn taking is defined is also expected given that these interventions contain aspects of both behavioral and developmental approaches (Odom et al., 2010). In addition to the variability in intervention approach, research designs varied across the studies we reviewed. Our search was not limited by research design and could have included qualitative studies, but research following qualitative designs was not identified. Qualitative research has numerous benefits to understanding social phenomena (Mohajan, 2018) and could enrich our knowledge of how children engage in turn taking through in-depth studies of the individual child and their communicative partner. Future qualitative investigations can further our insight into children's use of the different turn-taking functions. Most studies in this review ( $n$ $=8$ ) followed single subject designs, and five were randomized controlled trials. Although randomized controlled trials are often considered the "gold standard" in intervention research, studies with single subject designs have the potential to be evidence-based practices (Hume et al., 2021; What Works Clearinghouse, 2020). In the future, explorations of the literature should consider the efficacy of turn-taking interventions and which interventions may be considered evidence based to better inform practice.

While this review was not concerned with intervention results and effects, it identified other intervention content. Most children included in the interventions were male, an expected finding given the current prevalence estimates of autism (Maenner et al., 2020). This finding may also be attributed to sex and gender differences in meeting current diagnostic criteria (Wilson et al., 2016; Wood-Downie et al., 2021). All studies reported that children had or were at risk for autism; however, four studies did not report diagnostic focused assessment data (i.e., Kemp et al., 2019; Kim \& Clarke, 2015; Shire et al., 2020; Wang, 2017). Researchers should consider clear reporting of participant characteristics, such as formative assessment data at pre-intervention, which may provide further understanding of the study and can help to inform directions for future intervention research. Although a variety of settings and agents were identified across studies, most interventions were conducted in participants' homes and with some level of parental involvement. Additionally, most interventions sought social communication outcomes to some degree, indicating consistent awareness of the importance of supporting this competency in young children with autism. The DEC (2014), an internationally recognized organization that provides guidance for early childhood intervention and special education, recommends a series of practices when working with young children who have or who are at risk for disabilities. The DEC (2014) recommendations most relevant to the present literature review are family involvement, natural environments, and practices that promote social emotional development through active learning. Family-centered interventions recognize the family as important agents, experts, and decision-makers in their child's life (DEC, 2014; Wetherby \& Woods, 2008). A natural environment, most notably the home setting, is one in which children engage in everyday routines and activities and are most comfortable and familiar with (DEC, 2014; Wetherby \& Woods, 2008). In intervention, children should be actively engaged in their own learning and encouraged to initiate and 
respond to meaningful, social communication with others, such as may be found in a turn-taking routine (DEC, 2014; Wetherby \& Woods, 2008). This recommendation is especially relevant to children with autism given the core challenge they have in social communication. Many of the interventions reviewed in the present study meet the recommended practices of the DEC (2014) to some degree. However, only three studies, which defined social turn taking (i.e., Dawson et al., 2010; Green et al., 2017; Schertz, Odom, et al., 2018) most optimally incorporate the DEC (2014) recommended practices indicated above. Future turntaking interventions should endeavor to fully incorporate these recommended practices to optimally promote outcomes for young children who have or who are at risk for autism.

In sum, the results of the present study indicate that turn taking may serve different functions depending on how it is being promoted in intervention and the intervention approach being followed. Furthermore, there remains a need for eliminating ambiguity in defining turn taking by form and function when incorporated in interventions for children with autism and to align intervention content with developmentally appropriate practices. The study findings should be interpreted cautiously because of the limited number of articles that were included in the review. More exhaustive, systematic reviews of the literature that incorporate additional methods, such as manual screening of reference lists and searching numerous databases, may have resulted in the identification of other relevant studies. The current review also used limited search terminology to keep the scope narrow; however, search terms were carefully chosen for relevance to the present study. Additionally, the authors served as independent coders in the present study, which may increase risks for inter-rater bias, and including coders who do not serve as authors may decrease these risks. However, because authors commonly serve as coders in literature reviews (e.g., Kamenopoulou et al., 2021; Pennington et al., 2012), we see this as a minor limitation. Finally, because this review relies on how turn taking was defined by researchers in published articles, unpublished materials, such as intervention protocols and coding manuals, which may offer more insight into the intended turn-taking function of an intervention, could not be explored. Overall, this literature review provides valuable information on the current state of turn-taking components in intervention and potential areas of study in future research and practice.

\section{Declarations}

Acknowledgements: Not applicable.

Authors' contributions: KL conceived and designed the study, contributed to all portions of the manuscript, and conducted literature search, screening, and coding procedures as defined in the methodology. AS also conducted literature search, screening, and coding procedures, and contributed to all portions of the manuscript. All authors have read and approved the final submission.

Competing interests: The authors declare they have no competing interests.

Funding: This study was not funded.

\section{References}

Adamson, L., McArthur, D., Markov, Y., Dunbar, B., \& Bakeman, R. (2001). Autism and joint attention: Young children's responses to maternal bids. Applied Developmental Psychology, 22(4), 439-453. https://doi.org/10.1016/S0193-3973(01)00089-2

Alpert, C., \& Kaiser, A. (1992). Training parents as milieu language teachers. Journal of Early Intervention, 16(1), 31-52. https://doi.org/10.1177/105381519201600104

American Psychiatric Association. (2013). Diagnostic and statistical manual of mental disorders (5th ed). Arlington: American Psychiatric Publishing. https://doi.org/10.1176/appi.books.9780890425596

Bryson, S. E., Zwaigenbaum, L., McDermott, C., Rombough, V., \& Brian, J. (2008). The Autism Observation Scale for Infants: Sale development and reliability data. Journal of Autism and Developmental Disorders, 38(4), 731-738. https://doi.org/10.1007/s10803$\underline{007-0440-\mathrm{y}}$

Chiang, H. M., Soong, W. T., Lin, T. L., \& Rogers, S. (2008). Nonverbal communication skills in young children with autism. Journal of Autism and Developmental Disorders, 38(10), 1898-1906. https://doi.org/10.1007/s10803-008-0586-2

Clifford, S. M., \& Dissanayake, C. (2009). Dyadic and triadic behaviours in infancy as precursors to later social responsiveness in young children with autistic disorder. Journal of Autism and Developmental Disorders, 39(10), 1369-1380. https://doi.org/10.1007/s10803-009-0748-x 
Defining turn taking in intervention...

Cochet, H., \& Byrne, R. W. (2016). Communication in the second and third year of life: Relationships between nonverbal social skills and language. Infant Behavior and Development, 44, 189-198. https://doi.org/10.1016/j.infbeh.2016.07.003

Constantino, J. N., \& Gruber, C. P. (2005). The Social Responsiveness Scale. Torrance, CA: Western Psychological Services.

Cooper, H. (2010). Research synthesis and meta-analysis: A step-by-step approach (4th ed.). Los Angeles, CA: Sage.

Curcio, F. (1978). Sensorimotor functioning and communication in mute autistic children. Journal of Autism and Developmental Disorders, 8(3), 281-292. https://doi.org/10.1007/BF01539631

David, D. O., Costescu, C. A., Matu, S., Szentagotai, A., \& Dobrean, A. (2020). Effects of a robot-enhanced intervention for children with ASD on teaching turn-taking skills. Journal of Educational Computing Research, 58(1), 29-62. https://doi.org/10.1177/0735633119830344

Dawson, G., Rogers, S., Munson, J., Smith, M., Winter, J., Greenson, ... Barley, J. (2010). Randomized, controlled trial of an intervention for toddlers with autism: The Early Start Denver Model. Pediatrics, 125(1), e17-e23. https://doi.org/10.1542/peds.2009-0958

Delehanty, A. D., Stronach, S., Guthrie, W., Slate, E., \& Wetherby, A. (2018). Verbal and nonverbal outcomes of toddlers with and without autism spectrum disorder, language delay, and global developmental delay. Autism and Developmental Language Impairments, 3, 1-19. https://doi.org/10.1177/2396941518764764

Division for Early Childhood. (2014). DEC Recommended Practices. Council for Exceptional Children. Retrieved from http://www.decsped.org/recommendedpractices

Gengoux, G. W., Schapp, S., Burton, S., Ardel, C. M., Libove, R. A., Baldi, G., ... Hardan, A. Y. (2019). Effects of a parent-implemented developmental reciprocity treatment program for children with autism spectrum disorder. Autism, 23(3), 713-725. https://doi.org/10.1177/1362361318775538

Gillespie-Lynch, K., Sepeta, L., Wang, Y., Marshall, S., Gomez, L., Sigman, M., \& Hutman, T. (2012). Early childhood predictors of the social competence of adults with autism. Journal of Autism and Developmental Disorders, 42(2), 161-174. https://doi.org/10.1007/s10803-011-1222-0

Green, J., Pickles, A., Pasco, G., Bedford, R., Wan, M. W., Elsabbagh, M., ... \& the British Autism Study of Infant Siblings (BASIS) Team. (2017). Randomised trial of a parent-mediated intervention for infants at high risk for autism: Longitudinal outcomes to age 3 years. Journal of Child Psychology and Psychiatry, 58(12), 1330-1340. https://doi.org/10.1111/jcpp.12728

Greenspan, S., \& Wieder, S. (2006). Engaging autism: Using the floortime approach to help children relate, communication, and think. Jackson: Perseus Books.

Harrist, A. W., \& Waugh, R. M. (2002). Dyadic synchrony: Its structure and function in children's development. Developmental Review, 22(4), 555-592. https://doi.org/10.1016/S0273-2297(02)00500-2

Hume, K., Steinbrenner, J. R., Odom, S. L., Morin, K. L., Nowell, S. W., Tomaszewski, B., ... Savage, M. N. (2021). Evidence-based practices for children, youth, and young adults with autism: Third generation review. Journal of Autism and Developmental Disorders, 1-20. https://doi.org/10.1007/s10803-020-04844-2

Individuals with Disabilities Education Improvement Act of 2004, 20 U.S.C. § 2647 (2004).

Isaksen, J., \& Holth, P. (2009). An operant approach to teaching joint attention skills to children with autism. Behavioral Interventions, 24, 215-236. https://doi.org/10.1002/bin.292

Kamenopoulou, L., Ali, A., \& Ockelford, A. (2021). Multi-sensory impairment: Convenient label or recipe for confusion? A scoping review of research conducted in England (2001-20). Journal of Research in Special Educational Needs, 21(2), 98-110. https://doi.org/10.1111/1471-3802.12503

Kemp, C., Stephenson, J., Cooper, M., \& Hodge, K. (2019). The use of peer mediation and educator facilitation to promote turn taking in young children with autism spectrum disorder in inclusive childcare. Infants \& Young Children, 32(3), 151-171. https://doi.org/10.1097/IYC.0000000000000146

Kim, S. H., Bal, V. H., Benrey, N., Choi, Y. B., Guthrie, W., Colombi, C., \& Lord, C. (2019). Variability in autism symptom trajectories using repeated observations from 14 to 36 months of age. Journal of the American Academy of Child and Adolescent Psychiatry, 57(11), 837-848. https://doi.org/10.1016/j.jaac.2018.05.026

Kim, S., \& Clarke, E. (2015). Case study: An iPad-based intervention on turn-taking behaviors in preschoolers with autism. Behavioral Development Bulletin, 20(2), 253-264. https://doi.org/10.1037/h0101314

Klin, A., Lin, D. J., Gorrindo, P., Ramsay, G., \& Jones, W. (2009). Two-year-olds with autism orient to nonsocial contingencies rather than biological motion. Nature, 459(7244), 257-261. https://doi.org/10.1038/nature07868

Koegel, R. L., Schreibman, L., Good, A. B., Cerniglia, L., Murphy, C., \& Koegel, L. K. (1989). How to teach pivotal behaviors to autistic children. Santa Barbara: University of California.

Lee, K., \& Schertz, H. H. (2020). Brief report: Analysis of the relationship between turn taking and joint attention for toddlers with autism. Journal of Autism and Developmental Disorders, 50(7), 2633-2640. https://doi.org/10.1007/s10803-019-03979-1 


\section{Kwangwon LEE \& Ashley STAGGS}

Lord, C., Luyster, R., Gotham, K., \& Guthrie, W. (2012). Autism diagnostic observational schedule--toddler module manual. Los Angeles, CA: Western Psychological Services. https://doi.org/10.1007/978-1-4419-1698-3_2011

Lord, C., Rutter. M., DiLavore, P. C., Risi, S., Gotham, K., \& Bishop, S. L. (1999). ADOS: Autism diagnostic observation schedule. Manual. Los Angeles: Wester Psychological Services. https://doi.org/10.1037/t17256-000

Maenner, M., Shaw, K. A., Baio, J., Washington, A., Patrick, M. DiReinzo, M.,...Dietz, P. M. (2020). Prevalence of autism spectrum disorder among children aged 8 years-autism and developmental disabilities monitoring network, 11 sites, United States, 2016. MMWR Surveillance Summaries, 69(4), 1-12. https://doi.org/10.15585/mmwr.ss6904a1

McFadden, B., Kamps, D., \& Heitzman-Powell, L. (2014). Social communication effects of peer-mediated recess intervention for children with autism. Research in Autism Spectrum Disorders, 8(12), 1699-1712. https://doi.org/10.1016/j.rasd.2014.08.015

Mohajan, H. K. (2018). Qualitative research methodology in social sciences and related subjects. Journal of Economic Development, Environment and People, 7(1), 23-48. https://doi.org/10.26458/jedep.v7i1.571

Moriuchi, J. M., Klin, A., \& Jones, W. (2016). Mechanisms of diminished attention to eyes in autism. American Journal of Psychiatry, 174(1), 26-35. https://doi.org/10.1176/appi.ajp.2016.15091222

Mundy, P. (1995). Joint attention and social-emotional approach behavior in children with autism. Emotions in Developmental Psychopathology, 7(1), 63-82. https://doi.org/10.1017/S0954579400006349

Mundy, P. (2016). Autism and joint attention. New York: The Guilford Press.

Mundy, P., Delgado, C., Block, J., Venezia, M., Hogan, A., \& Seibert, J. (2003). Early social communication scales (ESCS). Coral Gables: University of Miami.

Mundy, P., Sigman, M., Ungerer, J., \& Sherman, T. (1986). Defining the social deficits of autism: The contribution of non-verbal communication measures. Journal of Child Psychology and Psychiatry, 27(5), 657-669. https://doi.org/10.1111/j.14697610.1986.tb00190.x

Odom, S. L., Boyd, B. A., Hall, L., J., \& Hume, K. (2010). Evaluation of comprehensive treatment models for individuals with autism spectrum disorders. Journal of Autism and Developmental Disorders, 40, 425-436. https://doi.org/10.1007/s10803-009-0825-1

Pennington, R. C., \& Delano, M. E. (2012). Writing instructions for students with Autism Spectrum Disorder: A review of the literature. Focus on Autism and Other Developmental Disabilities, 27(3), 158-167. https://doi.org/10.1177/1088357612451318

Poon, K. K., Watson, L. R., Baranek, G. T., \& Poe, M. D. (2012). To what extent do joint attention, imitation, and object play behaviors in infancy predict later communication and intellectual functioning in ASD?. Journal of Autism and Developmental Disorders, 42(6), 1064-1074. https://doi.org/10.1007/s10803-011-1349-z

Raulston, T. J., Hansen, S. G., Frantz, R., Machalicek, W., \& Bhana, N. (2020). A parent-implemented playdate intervention for young children with autism and their peers. Journal of Early Intervention, 42(4), 303-320. https://doi.org/10.1177/1053815119880943

Rieth, S. R., Stahmer, A. C., Suhrheinrich, J., Schreibman, L., Kennedy, J., \& Ross, B. (2014). Identifying critical elements of treatment: Examining the use of turn taking in autism intervention. Focus on Autism and Other Developmental Disabilities, 29(3), 168-179. https://doi.org/10.1177/1088357613513792

Rogers, S. J., Dawson, G., \& Vismara, L. A. (2012). An early start for your child with autism: Using everyday activities to help kids connect, communicate, and learn. New York: The Guilford Press.

Rogers, S., \& Wallace, K. (2011). Intervention for infants and toddlers with autism spectrum disorders. Autism Spectrum Disorders, 1081-1094. https://doi.org/10.1093/med/9780195371826.003.0069

Rollins, P. R., Campbell, M., Thibodeau Hoffman, R., \& Self, K. (2016). A community-based early intervention program for toddlers with autism spectrum disorders. Autism, 20(2), 219-232. https://doi.org/10.1177/1362361315577217

Schertz, H. H., Reichow, B., Tan, P., Vaiouli, P., \& Yildirim, E. (2012). Interventions for toddlers with autism spectrum disorders: An evaluation of research evidence. Journal of Early Intervention, 34(3), 166-189. https://doi.org/10.1177/1053815117737353

Schertz, H., H., Call-Cummings, M., Horn, K., Quest, K., \& Steffen Law, R. (2018). Social and instrumental interaction between parents and their toddlers with autism: A qualitative analysis. Journal of Early Intervention, 40(1), 20-38. https://doi.org/10.1007/s10803017-3386-8

Schertz, H., Odom, S., Baggett, K., \& Sideris, J. (2018). Mediating parent learning to promote social communication for toddlers with autism: Effects from a randomized controlled trial. Journal of Autism and Developmental Disorders, 48(3), 853-867. https://doi.org/10.1177/1053815112470721

Schopler, E., Van Bourgondien, M. E., Wellman, G. J., \& Love, S. R. (2010). Childhood Autism Rating Scale (2nd ed.). Los Angeles, CA: Western Psychological Services.

Shire, S. Y., Shih, W., Bracaglia, S., Kodjoe, M., \& Kasari, C. (2020). Peer engagement in toddlers with autism: Community implementation of dyadic and individual Joint Attention, Symbolic Play, Engagement, and Regulation intervention. Autism, 24(8), 2142-2152. https://doi.org/10.1177/1362361320935689 
Sigman, M., \& Ruskin, E. (1999). Nonverbal communication, play, and language skills. Monographs of the Society for Research in Child Development, 63(1), 29-53. https://doi.org/10.1111/1540-5834.00004

Therrien, M. C. S., \& Light, J. C. (2018). Promoting peer interaction for preschool children with complex communication needs and autism spectrum disorder. American Journal of Speech-Language Pathology, 27(1), 207-221. https://doi.org/10.1044/2017 AJSLP$\underline{17-0104}$

Thompson, G. A., McFerran, K. S., \& Gold, C. (2014). Family-centered music therapy to promote social engagement with severe autism spectrum disorder: A randomized controlled study. Child: Care, Health and Development, 40(6), 840-852. https://doi.org/10.1111/cch.12121

Trevarthen, C., \& Hubley, P. (1978). Secondary intersubjectivity: Confidence, confiding and acts of meaning in the first year. In A. Lock (Ed.), Action gesture and symbol (pp. 183-299). London: Academic Press.

Wang, H. T. (2017). Utilizing primary tier intervention to enhance reciprocal turn-taking of children with autism in Taiwan. Education and Training in Autism and Developmental Disabilities, 52(1), 64-76.

Wetherby, A. M., \& Woods, J. (2008). Developmental approaches to treatment. In K. Chawarska, A. Klin \& F. R. Volkmar (Eds.), Autism spectrum disorders in infants and toddlers: Diagnosis, assessment, and treatment (pp. 170-206) New York: The Guilford Press.

What Works Clearinghouse. (2020). What Works Clearinghouse standards handbook, version 4.1. U.S. Department of Education, Institute of Education Sciences, National Center for Education Evaluation and Regional Assistance.

Wilson, C., Murphy, C. M., McAlonan, G., Robertson, D. M., Spain, D., Hayward, H., .. Murphy, D. G. M. (2016). Does sex influence the diagnostic evaluation of autism spectrum disorder in adults?. Autism, 20(7), 808-819. https://doi.org/10.1177/1362361315611381

Wood-Downie, H., Wong, B., Kovshoff, H., Mandy, W., Hull, L., \& Hadwin, J. A. (2021). Sex/gender differences in camouflaging in children and adolescent with autism. Journal of Autism and Developmental Disorders, 51, 1353-1364. https://doi.org/10.1007/s10803-020-04615-Z 